\title{
openheart A comparative study of different imaging modalities for successful percutaneous left atrial appendage closure
}

Danny HF Chow, Gintautas Bieliauskas, Fadi J Sawaya, Oscar Millan-Iturbe, Klaus F Kofoed, Lars Søndergaard, Ole De Backer

\section{ABSTRACT}

- Additional material is published online only. To view please visit the journal online (http://dx.doi.org/10.1136/ openhrt-2017-000627)

To cite: Chow DHF, Bieliauskas G, Sawaya FJ, et al. A comparative study of different imaging modalities for successful percutaneous left atrial appendage closure. Open Heart 2017;4:e000627. doi:10.1136/ openhrt-2017-000627

Received 18 March 2017 Revised 20 April 2017 Accepted 9 May 2017

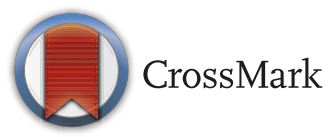

The Heart Center Rigshospitalet University Hospital, Copenhagen, Denmark

\section{Correspondence to}

Dr Danny HF Chow; danny.chow. hf@gmail.com
Objectives Accurate sizing of the left atrial appendage (LAA) is essential when performing percutaneous LAA closure. This study aimed to compare different LAA imaging modalities and sizing methods in order to obtain successful LAA closure.

Background Percutaneous LAA closure is an increasingly used treatment strategy to prevent stroke in patients with atrial fibrillation. LAA sizing has typically been done by 2D-transoesophageal echocardiography (TEE).

Methods Patients who had a preprocedural TEE and preprocedural and postprocedural multislice CT (MSCT) were identified. Preprocedural measurements of LAA ostia and landing zones by 2D-TEE, MSCT and angiography were collected and analysed for those patients with successful LAA closure - i.e. with no contrast leakage at 3-month follow-up MSCT.

Results The study population $(n=67)$ had a mean CHA DS - -VASc score of 3.0 and HAS-BLED score of 2.7. Fifty-eight patients (87\%) were identified to have successful LAA closure. Based on MSCT, 48 LAA sizings (83\%) resulted in a correct LAA closure device size selection, whereas with 2D-TEE sizing, only 33 measurements $(57 \%)$ would have resulted in a correct device size selection $(p<0.01)$. Using adapted BlandAltman method, MSCT-based perimeter-derived mean diameter was shown to be the best parameter to guide LAA device size selection for â€ closed-endâ€ $€^{\mathrm{TM}}$ devices (Amulet, WatchmanFLX), whereas the maximal diameter was the best parameter for the â€ open-endâ€ $€^{\mathrm{TM}}$ Watchman device.

Conclusions Preprocedural MSCT-based LAA closure device size selection proves to be a more accurate method than conventional 2D-TEE-based sizing. Depending on the LAA closure device design, perimeter-derived mean diameter or maximal diameter could be the better sizing method.

\section{INTRODUCTION}

Percutaneous left atrial appendage (LAA) closure is being increasingly used as a treatment strategy to prevent stroke in patients with atrial fibrillation (AF). The current indications for percutaneous LAA closure vary geographically. Recently, the European

\section{KEY QUESTIONS}

What is already known about this subject? Accurate sizing of the left atrial appendage (LAA) is essential when performing percutaneous LAA closure. 2D-transoesophageal echocardiography (TEE) is the officially recommended imaging modality in the company's instructions for use; however, other imaging modalities could also be used.

What does this study add?

Preprocedural multislice CT (MSCT)-based LAA closure device size selection proves to be a more accurate method than conventional 2D-TEE-based sizing, with a lower rate of residual LAA leakage. Depending on the LAA device design, MSCT-based perimeter-derived mean diameter is the best parameter to guide device size selection for 'closed-end' devices (Amulet/ WatchmanFLX), whereas maximal diameter is the best parameter for the 'open-end' Watchman device.

How might this impact on clinical practice? With a more accurate preprocedural LAA sizing, the next step will be to investigate whether preprocedural MSCT-based sizing in combination with intracardiac echocardiography-guided LAA device implantation could become the new standard, opening up the possibility for LAA closure under local anaesthesia.

Society of Cardiology confirmed a class IIB recommendation for patients with $\mathrm{AF}$ and contraindication(s) for long-term oral anticoagulant treatment. ${ }^{1}$ In the USA, the Watchman device (Boston Scientific, Massachusetts, USA) was approved by the Food and Drug Administration in 2015 for stroke prevention in patients with AF who have 'an appropriate rationale to seek a non-pharmacologic alternative to warfarin, taking into account the safety and effectiveness of the device compared to warfarin'.

In the 4-year follow-up from PROTECT-AF, patients with percutaneous LAA closure were found to have lower rates of haemorrhagic 
stroke as well as cardiovascular and all-cause mortality. ${ }^{2}$ Although these results are promising, percutaneous LAA closure carries some procedural risks such as cardiac perforation with pericardial effusion. Undersizing of the device may lead to significant peridevice leakage or even device embolisation, while oversizing of the device may cause tamponade or also device embolisation. ${ }^{34}$ In order to improve procedural and clinical outcomes, optimal LAA imaging and sizing is essential.

Although transoesophageal echocardiography (TEE) has been the main imaging modality for LAA assessment in most studies and centres, other imaging modalities such as multislice CT (MSCT) and intracardiac echocardiography (ICE) could be considered and are increasingly used.

In this retrospective study, we aimed to compare the accuracy of different LAA imaging and sizing modalities in order to obtain successful percutaneous LAA closure.

\section{METHODS}

\section{Study population}

In the period September 2014 to August 2016, 129 patients underwent percutaneous LAA closure at Rigshospitalet, Copenhagen University Hospital, Denmark. For this retrospective LAA imaging study, 67 patients who had a preprocedural TEE and MSCT as well as a postprocedural MSCT at 3 months after LAA closure were identified. All patients were fully informed about the procedure and provided informed written consent for the procedure and data collection. As a routine, preprocedural TEE and MSCT were done as outpatient procedures and 500 $\mathrm{mL}$ of saline infusion were given to patients before TEE, MSCT and LAA closure to ensure adequate hydration and standardised conditions for LAA sizing.

\section{Transoesophageal echocardiography}

Two-dimensional (2D) TEE was performed using a commercially available TEE transducer (Vivid, Philips, the Netherlands). The maximal LAA ostium and landing zone widths were measured in the mid-oesophageal view at $0^{\circ}, 45^{\circ}, 90^{\circ}$ and $135^{\circ}$ angles, according to company recommendations. For the Watchman devices (Boston Scientific), the maximal LAA ostium width was measured from the coronary artery marker to a point $2 \mathrm{~cm}$ from the tip of the 'limbus' at the $0^{\circ}$ angle and from the top of the mitral valve annulus to a point $2 \mathrm{~cm}$ from the tip of the 'limbus' at the other angles. For the Amulet LAA occluder (St Jude Medical, Minnesota, USA), the maximal landing zone width was measured $10 \mathrm{~mm}$ distally from the LAA ostium at the level of the left circumflex artery. ${ }^{4}$

\section{Multislice CT}

MSCT imaging was performed using a 320-multidetector scanner (Aquilion One Vision Edition, Toshiba Medical Systems, Japan). Contrast phase was reconstructed in $0.5 \mathrm{~mm}$ slice thickness with $0.25 \mathrm{~mm}$ increment; non-contrast phase was reconstructed in $3.0 \mathrm{~mm}$ slice thickness with $3.0 \mathrm{~mm}$ increment (see online supplementary file S1).

All MSCT images were analysed using the commercially available Osirix software package. Preprocedural LAA assessment and sizing was performed by $3 \mathrm{D}$ multiplanar reconstruction (MPR) using the end-diastolic phase series. 3D MPR allows assessment of the LAA anatomy in three different planes (sagittal, coronal and axial) locked at $90^{\circ}$ angles. LAA morphology was classified into windsock, chicken wing, cactus and cauliflower. The LAA ostium and landing zone for the different devices were identified and the minimal diameter, maximal diameter and perimeter of these axial LAA views were measured.

All patients included in this study also received a control MSCT scan at 3 months postprocedure for assessment of LAA closure device position, device compression,

Table 1 Baseline characteristics of study population $(n=67)$

Patient characteristics

\begin{tabular}{|c|c|}
\hline Age, years & $74 \pm 7$ \\
\hline Female & $36(54 \%)$ \\
\hline Hypertension & $41(61 \%)$ \\
\hline Diabetes mellitus & $16(24 \%)$ \\
\hline Stroke/TIA/thromboembolism & $37(55 \%)$ \\
\hline Vascular disease & $11(16 \%)$ \\
\hline Congestive heart failure & $5(7 \%)$ \\
\hline Abnormal liver function & $1(1 \%)$ \\
\hline Abnormal renal function & $9(13 \%)$ \\
\hline Labile INRs & $2(3 \%)$ \\
\hline Indication for antithrombotic medication & $17(25 \%)$ \\
\hline Alcohol & $3(4 \%)$ \\
\hline $\mathrm{CHA}_{2} \mathrm{DS}_{2}$-VASc score & $3.0 \pm 1.6$ \\
\hline HAS-BLED score & $2.7 \pm 0.9$ \\
\hline \multicolumn{2}{|l|}{ Procedural characteristics } \\
\hline \multicolumn{2}{|l|}{ Anaesthesia/intraprocedural imaging } \\
\hline G.A. with TEE guidance & $28(42 \%)$ \\
\hline L.A. with ICE guidance & $39(57 \%)$ \\
\hline \multicolumn{2}{|l|}{ LAA closure device } \\
\hline Amulet & $44(66 \%)$ \\
\hline Watchman & $11(16 \%)$ \\
\hline WatchmanFLX & $12(18 \%)$ \\
\hline Procedure time (min) & $46 \pm 13$ \\
\hline Contrast volume (mL) & $135 \pm 42$ \\
\hline \multicolumn{2}{|l|}{ Postprocedural medication } \\
\hline Single antiplatelet agent & $4(6 \%)$ \\
\hline Double antiplatelet therapy & $63(94 \%)$ \\
\hline Oral anticoagulation & - \\
\hline
\end{tabular}

G.A, general anaesthesia; ICE, intracardiac

echocardiography; INR, international normalised ratio; L.A, local anaesthesia; LAA, left atrial appendage; TEE, transoesophageal echocardiography; TIA, transient ischaemic attack. 


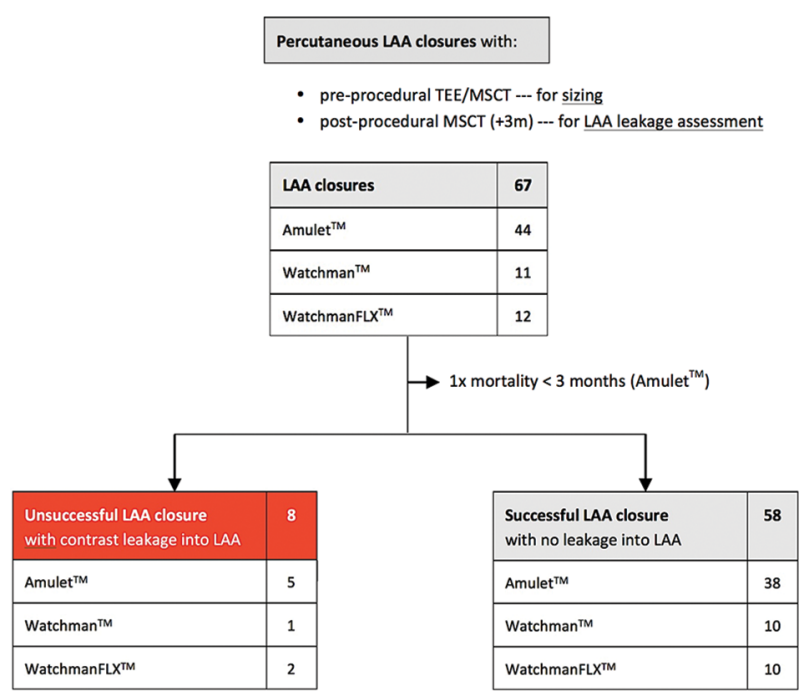

Figure 1 Flow chart. Flow chart showing the number of patients with successful percutaneous LAA closure based on the evaluation of the 3-month CT follow-up. LAA, left atrial appendage; MSCT, multislice CT; TEE, transoesophageal echocardiography.

peridevice leakage and device-related thrombosis. In case of no contrast leakage into the LAA, the LAA closure was considered successful.

\section{Percutaneous LAA closure procedure}

Percutaneous LAA closures were performed either under general anaesthesia with TEE guidance or with local
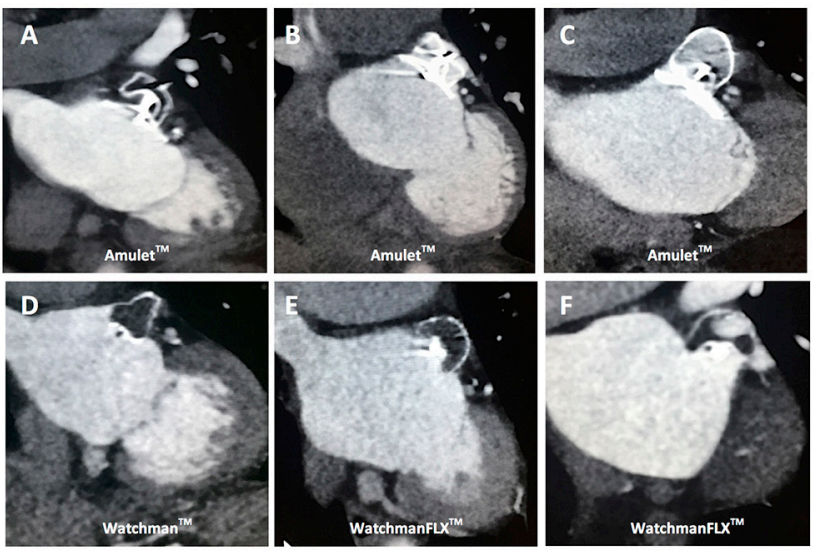

Figure 2 Postprocedural multislice CT (MSCT) followup. MSCT control scans at 3 months postprocedure to assess LAA device position and complete LAA closure. (A) Successful LAA closure with an Amulet occluder with no contrast leakage into LAA. (B) Unsuccessful LAA closure with contrast leakage into LAA due to Amulet device undercompression. (C) Unsuccessful LAA closure with contrast leakage into LAA due to Amulet device overcompression. (D) Successful LAA closure with a Watchman LAA closure device with no contrast leakage into LAA. (E) Successful LAA closure with a WatchmanFLX LAA closure device with no contrast leakage into LAA. (F) Unsuccessful LAA closure with contrast leakage into LAA due to a rotated, non-occlusive WatchmanFLX closure device. LAA, left atrial appendage. anaesthesia and ICE guidance. Following routine transseptal puncture (posterior and slightly inferior), the LAA was closed by one of the following three LAA closure devices: Amplatzer Amulet LAA occluder, Watchman or WatchmanFLX device, as described previously. ${ }^{45}$

\section{Statistical analysis}

Continuous data are reported as mean $\pm \mathrm{SD}$, and categorical variables are reported as number of patients and percentages. Categorical data were compared using Fisher's exact test, and continuous data using Student's t-test or Mann-Whitney's U test, as appropriate. An adapted Bland-Altman method was used to investigate the accuracy of the different imaging modalities in order to obtain successful LAA closure with no contrast leakage into the LAA - the 95\% limits of agreement (mean difference $\pm 2 \mathrm{xSD}$ ) was calculated for every specific LAA closure device and imaging modality. All tests were two sided, and $\mathrm{p}$ values $<0.05$ were considered to be statistically significant. All analyses were conducted using SPSS statistical software V. 23.0 (IBM, Armonk, New York, USA).

\section{RESULTS \\ Baseline characteristics}

A total of 67 patients were included in this study. The mean age of the patient cohort was 74 years and 36 patients $(54 \%)$ were female. The average $\mathrm{CHA}_{2} \mathrm{DS}_{2}-$ VASc score was 3.0 and HAS-BLED score was 2.7. Three different LAA closure devices were used: Amulet $(n=44$; $66 \%)$, Watchman $(\mathrm{n}=11 ; 16 \%)$ and WatchmanFLX $(\mathrm{n}=12 ; 18 \%)$. The majority of patients $(\mathrm{n}=63 ; 94 \%)$ were treated with dual antiplatelet therapy during the first 3 months postimplantation (table 1). A list of indications for percutaneous LAA closure can be found in online supplementary file S2.

\section{Successful LAA closure}

Of all 67 patients who underwent percutaneous LAA closure and who were planned for a control CT scan after 3 months, 1 patient died within 3 months and 8 other patients were found to have contrast leakage into the LAA (figure 1). From the three patients with $<10 \%$ of device compression after implantation, two patients $(67 \%)$ had contrast leakage at control CT scan. In the group of patients with $10 \%-20 \%$ of LAA device compression, only one patient $(1 / 38,3 \%)$ had unsuccessful LAA closure with contrast leakage into the LAA. From those with $>20 \%$ device compression, five patients $(5 / 25,20 \%)$ had contrast leakage into the LAA (figure 2 and 3 ).

\section{LAA sizing by different imaging modalities}

As those cases with a completely occluded LAA without residual LAA leakage can be considered truly successful LAA closures, the LAA closure device sizes used in these cases can be considered as the 'optimal' device size selection for these specific cases. Hence, further analysis was done on patients with successful LAA closure $(n=58)$, i.e. with no contrast leakage at 3-month CT follow-up. 


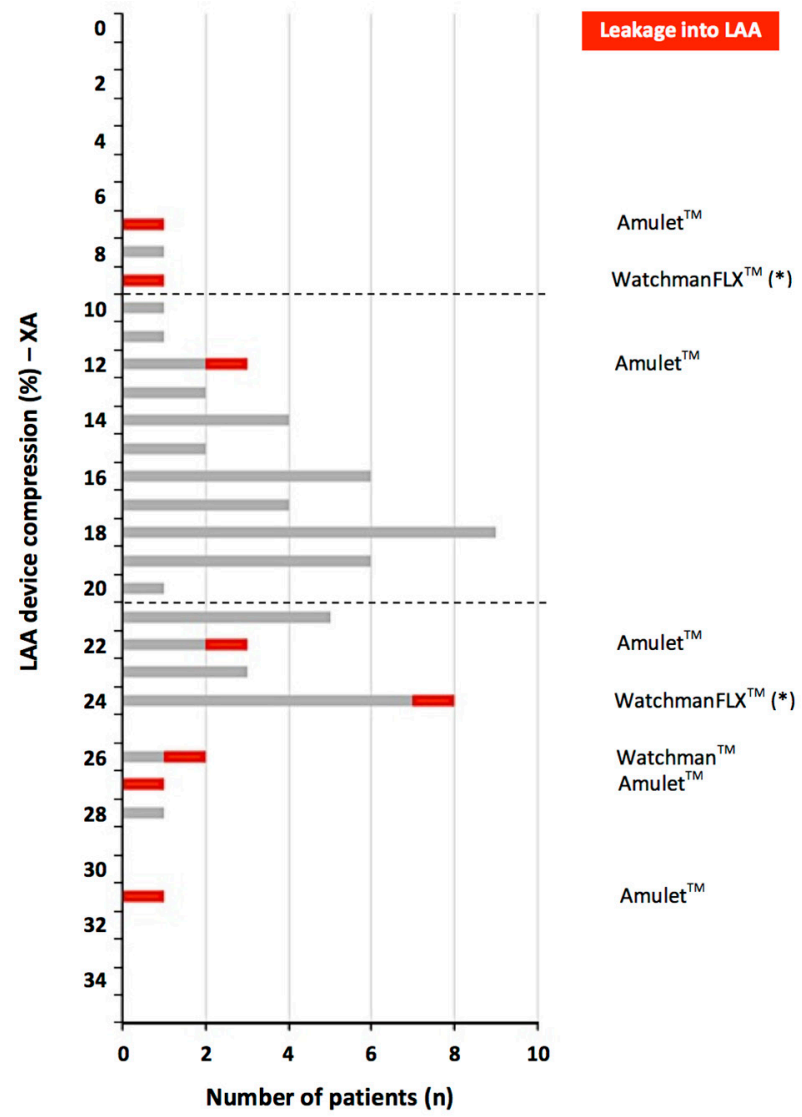

Figure 3 LAA device compression and LAA leakage. Correlation between LAA closure device compression rate (\%, as assessed on angiography postimplantation) and incidence of LAA leakage as observed at 3 months multislice CT follow-up. In case of 10\%-20\% device compression, successful LAA closure was obtained in 37 out of 38 cases (97\%); leakage into the LAA was more frequently observed in case of LAA device undercompression or overcompression. LAA, left atrial appendage.

The differences between the obtained LAA sizings with the different imaging modalities and the used LAA closure device size-in millimetre-were calculated for every single case. Based on these calculations, the mean difference and $95 \%$ limits of agreement (LoA) could be calculated for each combination of LAA closure device and sizing method. The LoA width was the narrowest for CT-based LAA sizing methods, namely 'CT-perimeter derived (PD) mean diameter' for the Amulet $(\Delta 5.6 \mathrm{~mm})$ and WatchmanFLX device $(\Delta 6.0 \mathrm{~mm})$, and 'CT-max diameter' for the Watchman device $(\Delta 5.2 \mathrm{~mm})$. These widths were narrower than 2D-TEE and angio-derived values, indicating that CT-based sizing methods were the most accurate (figure 4).

Figure 5 shows Bland-Altman plots for the three different LAA closure devices used in this study and the results obtained with 2D-TEE and the most optimal MSCTbased sizing method. Based on the company-derived LAA sizing recommendations (see online supplementary file S3) and the MSCT-based recommendation to select the first-available larger LAA device size ('one step up'; see online supplementary file S4), the number of correct LAA device size selections per imaging modality could be calculated. Based on TEE sizing, only 33 out of 58 LAA sizings $(57 \%)$ would have led to the correct LAA closure device size, whereas in case of MSCT-based sizing, 48 out of 58 LAA sizings ( $83 \%$ ) would have resulted in the correct device size selection $(\mathrm{p}<0.01$; figure 5$)$.

Finally, we can report that LAA sizing by ICE was attempted in 10 patients; however, due to the impossibility to assess the LAA in multiple planes and the incorrect LAA sizings obtained in these 10 patients (data not shown), this LAA sizing modality was not further explored. As a result, ICE was solely used as a guidance tool for safe transseptal puncture and evaluation of successful LAA closure ad hoc.

\section{DISCUSSION}

Percutaneous LAA closure is an increasingly used treatment strategy to prevent stroke in patients with AF. This study aimed to compare the accuracy of different LAA imaging modalities and sizing methods in order to optimise procedural outcomes.

From experience in the field of transcatheter aortic valve replacement (TAVR), it was learnt that aortic annulus sizing by 2D echocardiography results in systematic underestimation of the true aortic annular diameter. In comparison, CT-based sizing allows a more precise assessment of the annulus and, hence, a more accurate transcatheter heart valve size selection for TAVR. ${ }^{6} \mathrm{Com}-$ pared with the aortic annulus, the LAA is an even more complex structure with a wide range of LAA shapes and sizes. ${ }^{7}$ Despite evaluation of the maximal LAA diameter at multiple angles by 2D-TEE, the measured maximal

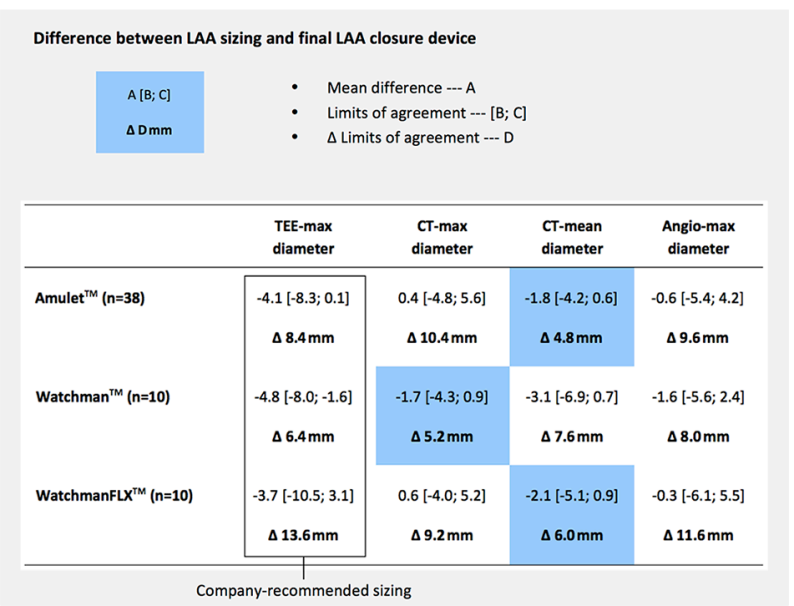

Figure 4 LAA sizing by different imaging modalities. The calculated mean difference between LAA sizing and final LAA closure device size for the different LAA closure devices and imaging modalities. This analysis only included successful percutaneous LAA closures $(n=58)$. Adapted Bland-Altman method was used to calculate the mean difference, $95 \%$ limits of agreement and $\Delta$ limits of agreement. LAA, left atrial appendage; TEE, transoesophageal echocardiography. 
(A) Successful LAA closure with Amulet ${ }^{\mathrm{TM}}$ device $(\mathrm{n}=38)$

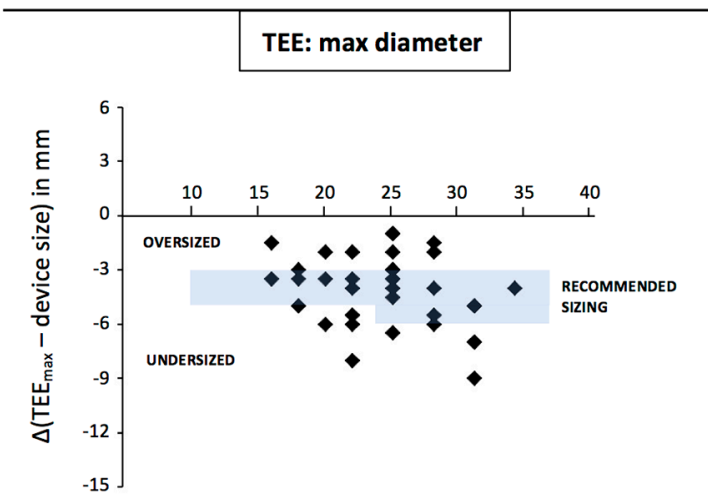

Correct sizing: 22/38 (58\%)

Limits of Agreement: [-8.3;0.1]: $\Delta \mathbf{8 . 4 m m}$

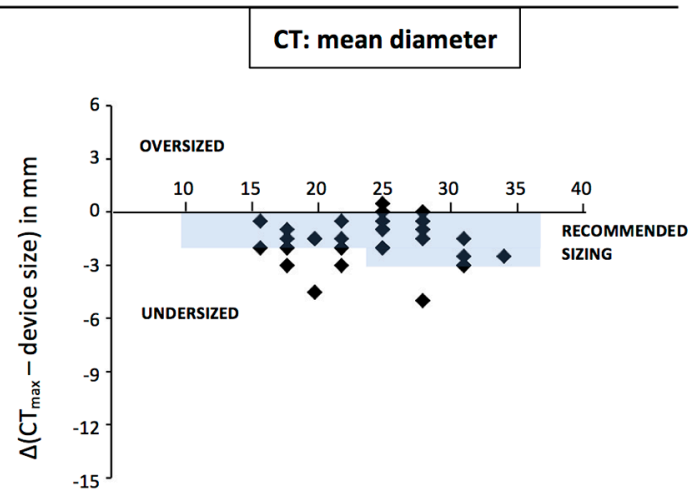

Correct sizing: $31 / 38$ (82\%)

Limits of Agreement: $[-4.2 ; 0.6]: \Delta 4.8 \mathrm{~mm}$

(B) Successful LAA closure with Watchman ${ }^{\text {TM }}$ device $(n=10)$

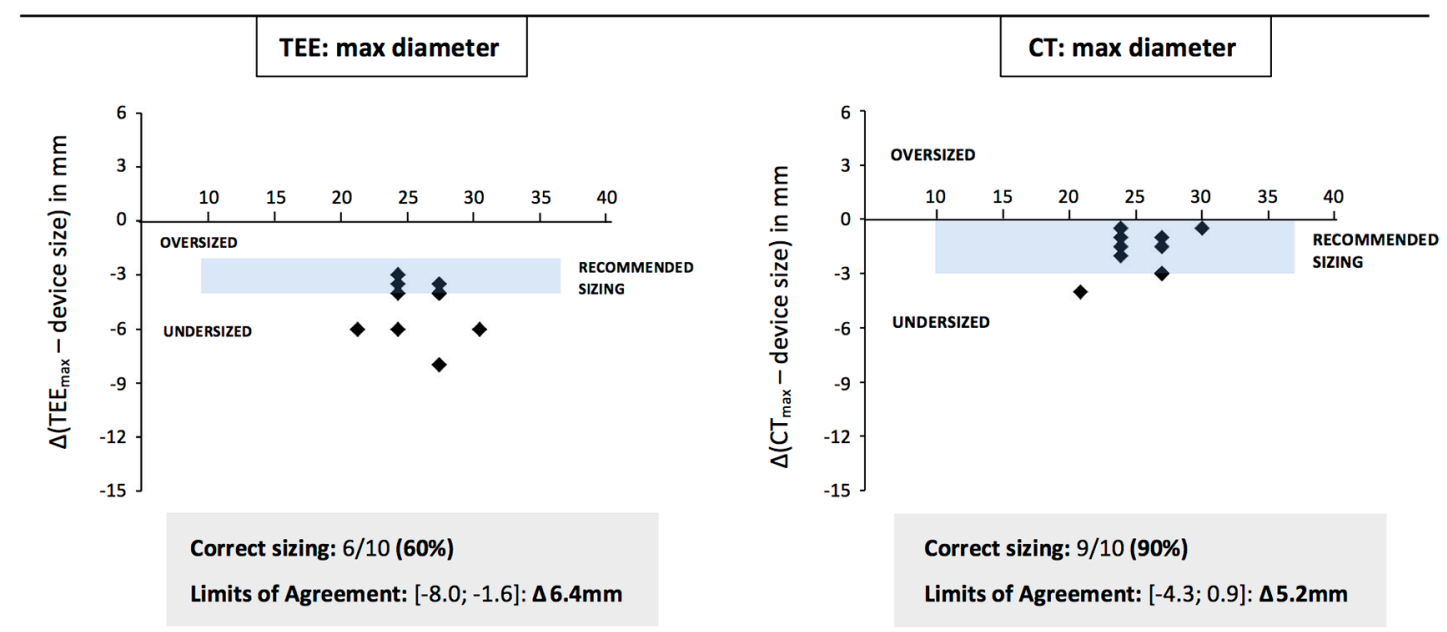

(C) Successful LAA closure with WatchmanFLX ${ }^{\text {TM }}$ device $(n=10)$

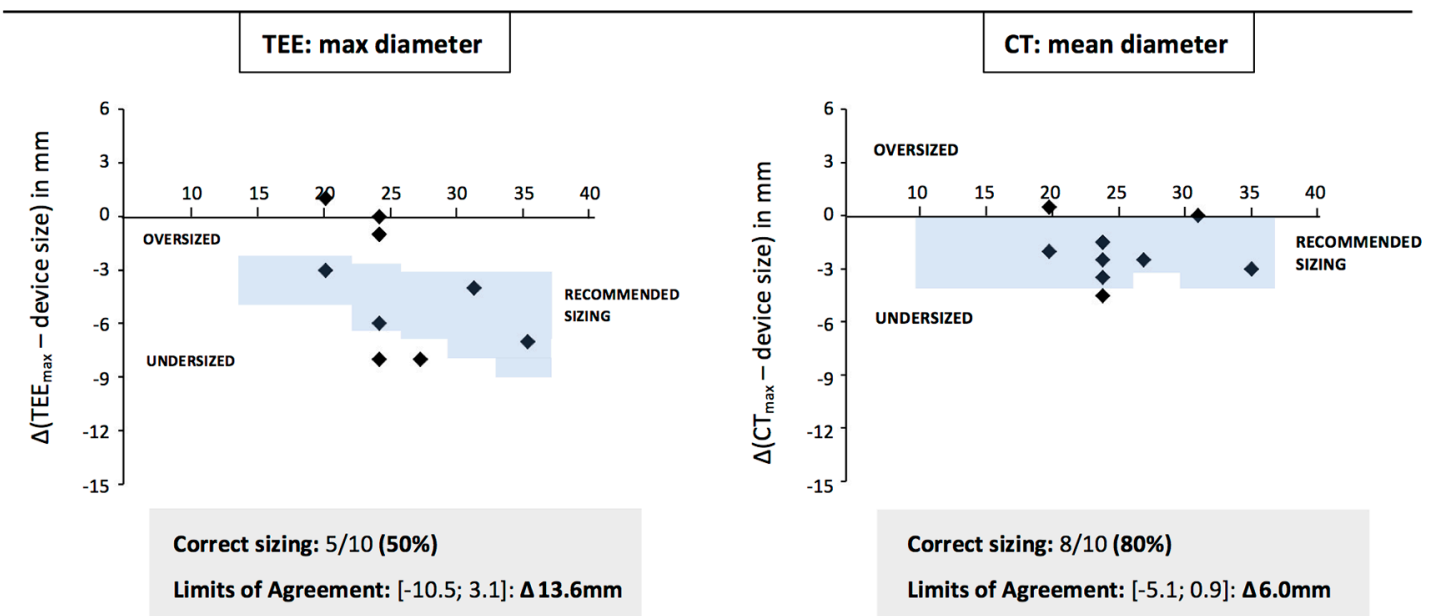

Figure 5 Bland-Altman plots. The adapted Bland-Altman plots for 2D-TEE and optimal CT-guided LAA closure device sizing. This analysis only included successful percutaneous LAA closures $(n=58)$. The plots show the difference between LAA sizing and final LAA closure device size for the different LAA closure devices and imaging modalities. Considering the official TEE-recommended sizing versus CT-based recommendation to select the first-available larger LAA device size ('one step up'), the number of correct sizings per device and imaging modality could be calculated. LAA, left atrial appendage; TEE, transoesophageal echocardiography. 
diameter may still underestimate the actual maximal LAA diameter (figure 6). This was also reported in a study by Shah $e t a l$ showing that 2D-TEE underestimates the LAA orifice area compared with 3D-TEE imaging. LAA orifice area as measured on cardiac MSCT was well correlated with LAA orifice area as measured by 3D-TEE but not by 2D-TEE. However, both 2D-TEE and 3D-TEE require the availability of a skilled echocardiographer ${ }^{9}$ and result in a greater interoperator variability for LAA sizing as compared with MSCT in our experience. The use of CT-3D MPR allows a more precise assessment of LAA morphology, the relationship between LAA and its surrounding structures, and a more accurate measurement of the LAA ostium and landing zone.

Despite growing interest in the application of CT imaging in preprocedural LAA closure planning, there is still no general consensus on how to guide LAA device size selection based on CT imaging. By using preprocedural and postprocedural CT scans, we were able to derive a general guidance for LAA device size selection (see online supplementary file S4) based on the criterion of 'successful LAA closure' without contrast leakage into the LAA at 3 months postprocedure. This is important since immediate 'procedural success' does not always lead to actual 'successful LAA closure', as observed at follow-up CT scan. The definition of successful 'LAA closure' in the PROTECT-AF trial was any seal with $\leq 3 \mathrm{~mm}$ width $( \pm 2 \mathrm{~mm})$ of peridevice flow. Of the 389 patients who had 12-month TEE follow-up, 125 patients (32\%) had persistent flow around the device and 46 $(11.8 \%)$ of them had major leakage $>3 \mathrm{~mm} .{ }^{10}$ Although there is currently no clinical evidence on the association between peridevice leakage and the risk of thromboembolism, a stricter definition of 'successful LAA closure' will be imperative to further improve clinical outcomes and broaden the acceptance of this procedure by a larger group of referring physicians.

Results in this study indicate that $10 \%-20 \%$ device compression immediately after implantation should be targeted in order to obtain good rates of 'successful LAA closure' at long-term follow-up. This is in alignment with the company-based recommendations for the Watchman devices (see online supplementary file S3); however, a company-recommended Amulet lobe compression rate is currently not available.

The data in this study also indicate that CT-based LAA sizing may be a more accurate method than 2D-TEEbased and/or angiographic LAA sizing. Although there may be concerns that using MSCT as a preprocedural planning tool may potentially lead to extra radiation and contrast exposure as compared with TEE, the improved LAA sizing accuracy obtained with MSCT may shorten the actual LAA closure procedure time, radiation exposure, decrease the number of LAA closure devices and volume of contrast used during the procedure ${ }^{11}$ Based on CT sizing, $80 \%-90 \%$ of LAA sizings resulted in the correct LAA closure device size selection, whereas in case of 2D-TEE-based sizing, only $50 \%-60 \%$ of LAA sizings

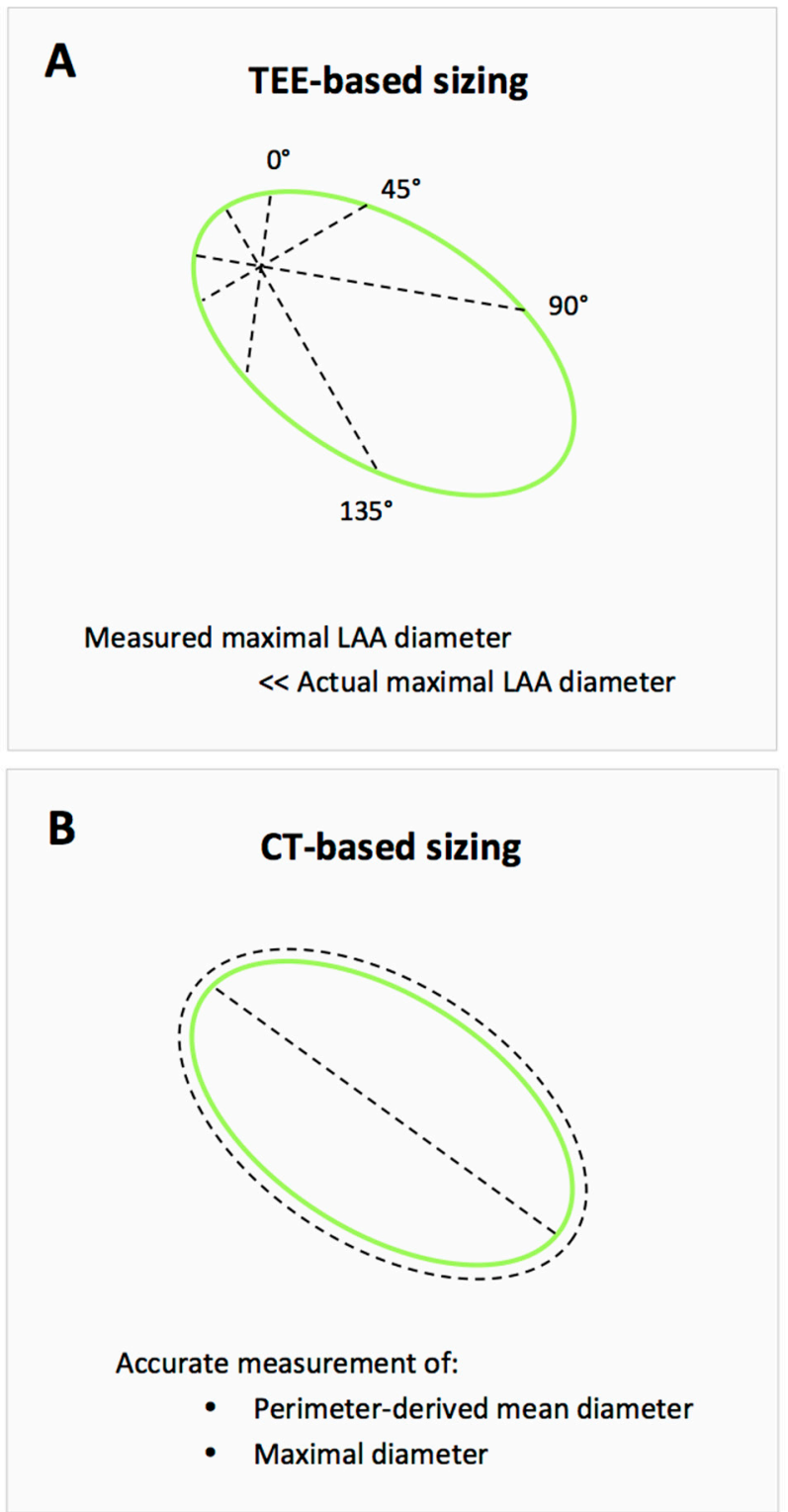

Figure 6 Challenges with 2D-TEE-based LAA sizing. (A) The disadvantage of 2D-TEE-guided sizing despite the use of multiple angles for assessment of maximal LAA diameter. (B) 3D MPR assessment from MSCT allows accurate measurement of the perimeter-derived mean diameter and maximal LAA diameter. LAA, left atrial appendage; MSCT, multislice CT; TEE, transoesophageal echocardiography.

would have led to correct LAA device size selection $(\mathrm{p}<0.01$; figure 5$)$.

Until now, conventional TEE-guided device size selection has been a uniform 'oversizing approach' based on the maximum LAA ostium width in case of Watchman devices and maximum LAA landing zone width in case of the Amulet device. However, these different closure devices have different designs and structures. The behaviour and deployment of these devices may vary, especially in elliptical LAA anatomies. For LAA closure devices with a 'closed' nitinol mesh design at its distal end, device compression in one plane results in device expansion in a 

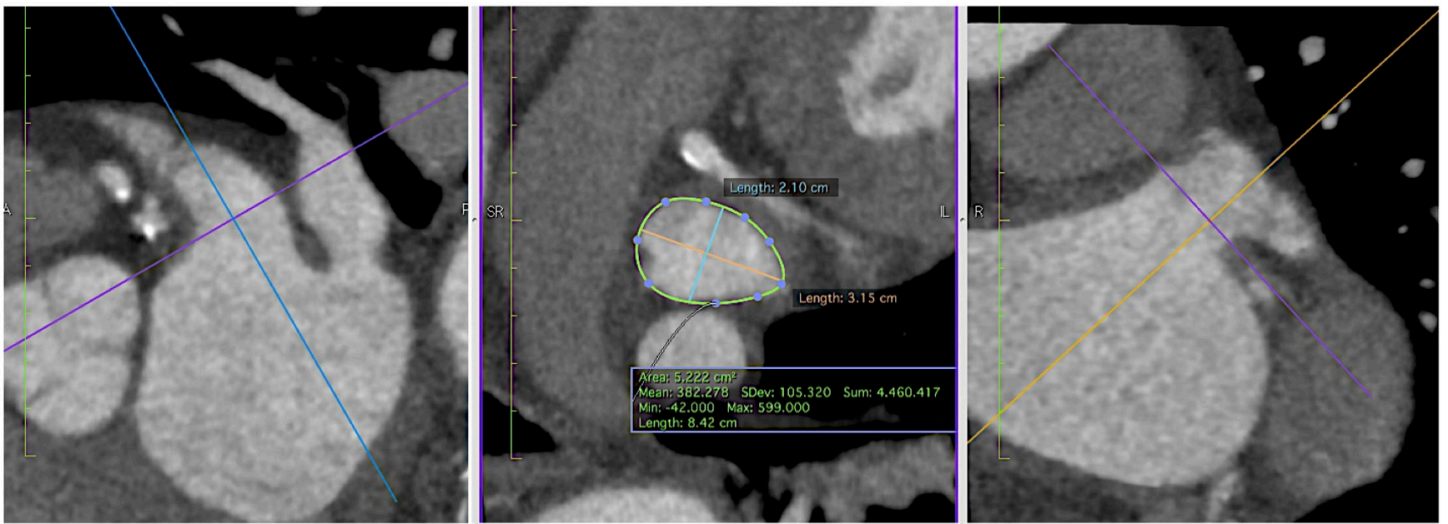

(A) CT-based sizing for “closed distal end" LAA closure devices (Amulet ${ }^{\mathrm{TM}}$, WatchmanFLX ${ }^{\mathrm{TM}}$ )
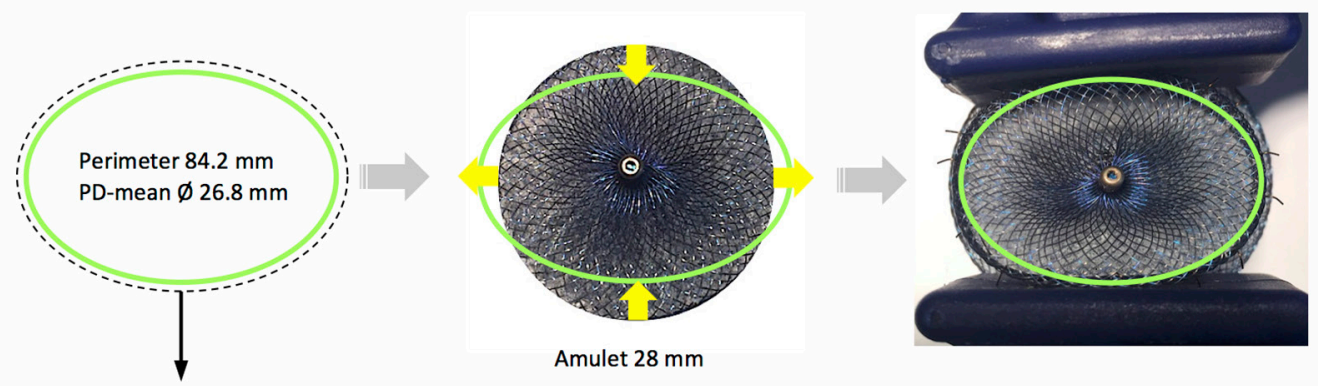

Perimeter-derived mean LAA diameter $\rightarrow$ select the first-available "larger" LAA device size ("one step up")-Amulet $28 \mathrm{~mm}$

(B) CT-based sizing for "open distal end" LAA closure devices (Watchman ${ }^{\mathrm{TM}}$ )
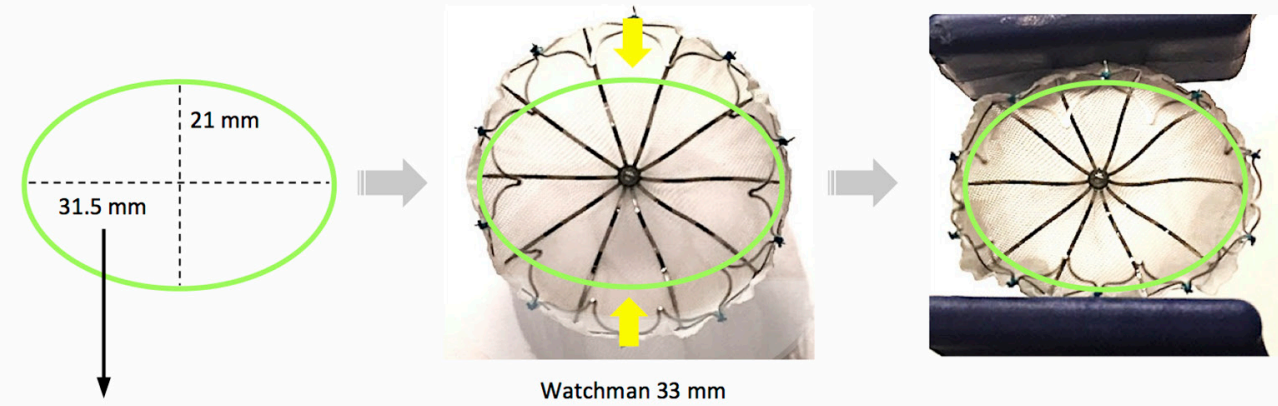

Maximal LAA diameter $\rightarrow$ select the first-available "larger" LAA device size ("one step up") - Watchman $33 \mathrm{~mm}$

Figure 7 Device-specific CT-based LAA occluder size selection. Preprocedural cross-sectional CT assessment of the LAA landing zone. Since the cross section of the LAA landing zone is more often an ellipse, the major axis (maximal diameter) and minor axis (minimal diameter) may differ. As the device comes first into contact with the short axis of the LAA during deployment, there will be a compressive force onto the device. Depending on the design of the closure device, the device will expand or not in a perpendicular plane to the short axis in case of a 'closed distal end' or 'open distal end' LAA closure device, respectively. LAA, left atrial appendage.

perpendicular plane. On the other hand, for LAA closure devices with an 'open' nitinol mesh design at its distal end, device compression in one plane does not have any effect on the device size in a perpendicular plane (figure 7). For this reason, CT-based LAA closure device size selection should differ for the Amulet and WatchmanFLX — which are 'closed-end' devices-from that of the Watchman, which is an 'open-end' closure device. The calculated LoA indicate that for 'closed-end' LAA closure devices, the best parameter for LAA device size selection is PD mean diameter, whereas for 'open-end' devices, the best parameter seems to be the maximal LAA diameter. The latter finding also indicates that sizing for the 'open-end' Watchman device is most likely more forgiving. When implanting 'closed-end' devices, too liberal oversizing may result in severe device underexpansion and, hence, a lot of 'stored energy' in the device, thereby increasing the risk of device embolisation. This 
difference in device characteristics and LAA sizing may have been unknown or ignored with the launch of the WatchmanFLX device, resulting in an excess number of device embolisations.

Finally, we would like to comment on the advantages and limitations connected with the use of ICE guidance during the procedure. First, by using ICE only during the procedure, this approach has created more flexibility in our scheduling as we plan LAA closure procedures without the need for an anaesthesiologist and/or TEE expert. Procedures can be performed with local anaesthesia only, without the need for sedative medication. Clearly, a disadvantage is the cost of the ICE probe which substantially adds extra costs. Another issue with ICE is the limitation to assess peridevice leakage in multiple planes after implantation. However, as supported by recent data reported by Bieliauskas et al, ${ }^{11}$ the incidence of residual LAA leakage was extremely low in case of preprocedural LAA sizing with MSCT and periprocedural ICE guidance.

\section{LIMITATIONS}

An obvious limitation of this retrospective study is the relatively limited number of patients included. Also the fact that both imaging tools were available to the operator before the actual procedure may have influenced the final LAA closure device size selection. Moreover, 3D-TEE data were not available. The unique aspect of this study is, however, that both preprocedural and postprocedural MSCT was available for all included patients, making the definition of successful LAA closure more solid.

\section{CONCLUSIONS}

This study indicates that MSCT-based LAA device size selection may be a more accurate method than conventional TEE-based sizing. Depending on the LAA device design, maximal or PD mean diameter could be the better sizing method. In addition, 10\%-20\% device compression postimplantation should be targeted in order to obtain complete LAA closure at long-term follow-up.

Contributors DHFC, ODB: study concept and design, drafting of manuscript,statistical analysis. DHFC, ODB, OMI, FJS: acquisition, analysis or interpretation of data. ODB, FJS, GB, KFK, LS: critical revision of manuscript. ODB: study supervision.

Competing interests None declared.

Patient consent Patient signed consent for this retrospective trial in danish.
Provenance and peer review Not commissioned; externally peer reviewed.

Data sharing statement Based on unique data provided by systematic preprocedural and postprocedural multislice CT (MSCT) imaging, this study indicates that preprocedural MSCT-based left atrial appendage (LAA) closure device size selection is more accurate than conventional 2D-transoesophageal echocardiography-based sizing. In addition, this study encompasses three different types of LAA closure devices and shows that, depending on the LAA device design, perimeter-derived mean diameter or maximal diameter could be the better sizing method. In conclusion, these data will be of high importance for the field of percutaneous LAA closure and will help to obtain even better procedural and clinical outcomes in the future.

Open Access This is an Open Access article distributed in accordance with the Creative Commons Attribution Non Commercial (CC BY-NC 4.0) license, which permits others to distribute, remix, adapt, build upon this work non-commercially, and license their derivative works on different terms, provided the original work is properly cited and the use is non-commercial. See: http://creativecommons.org/ licenses/by-nc/4.0/

(C) Article author(s) (or their employer(s) unless otherwise stated in the text of the article) 2017. All rights reserved. No commercial use is permitted unless otherwise expressly granted.

\section{REFERENCES}

1. Kirchhof P, Benussi S, Kotecha D, et al. 2016 ESC Guidelines for the management of atrial fibrillation developed in collaboration with EACTS. Eur Heart J 2016;37:2893-962.

2. Reddy VY, Sievert H, Halperin J, et al. Percutaneous left atrial appendage closure vs warfarin for atrial fibrillation: a randomized clinical trial. JAMA 2014;312:1988-98.

3. Onalan O, Crystal E. Left atrial appendage exclusion for stroke prevention in patients with nonrheumatic atrial fibrillation. Stroke 2007;38:624-30.

4. De Backer O, Arnous S, Ihlemann N, et al. Percutaneous left atrial appendage occlusion for stroke prevention in atrial fibrillation: an update. Open Heart 2014;1:e000020.

5. Saw J, Lempereur M. Percutaneous left atrial appendage closure: procedural techniques and outcomes. JACC Cardiovasc Interv 2014;7:1205-20.

6. Kempfert J, Van Linden A, Lehmkuhl L, et al. Aortic annulus sizing: echocardiographic versus computed tomography derived measurements in comparison with direct surgical sizing. Eur $J$ Cardiothorac Surg 2012;42:627-33.

7. Kerut EK. Anatomy of the left atrial appendage. Echocardiography 2008;25:669-73.

8. Shah SJ, Bardo DM, Sugeng L, et al. Real-time three-dimensional transesophageal echocardiography of the left atrial appendage: initial experience in the clinical setting. J Am Soc Echocardiogr 2008;21:1362-8.

9. Wunderlich NC, Beigel R, Swaans MJ, et al. Percutaneous interventions for left atrial appendage exclusion: options, assessment, and imaging using $2 \mathrm{D}$ and $3 \mathrm{D}$ echocardiography. JACC Cardiovasc Imaging 2015;8:472-88.

10. Viles-Gonzalez JF, Kar S, Douglas P, et al. The clinical impact of incomplete left atrial appendage closure with the Watchman Device in patients with atrial fibrillation: a PROTECT AF (Percutaneous closure of the left Atrial Appendage Versus Warfarin therapy for Prevention of Stroke in Patients with Atrial Fibrillation) substudy. $J$ Am Coll Cardiol 2012;59:923-9.

11. Bieliauskas G, Otton J, Chow DHF, et al. Use of 3-Dimensional Models to Optimize Pre-Procedural Planning of Percutaneous Left Atrial Appendage Closure. JACC Cardiovasc Interv 2017;10:1067-70. 